\title{
PROSPECTIVE HEALTHCARE DECISION-MAKING BY COMBINED SYSTEM DYNAMICS, DISCRETE-EVENT AND AGENT-BASED SIMULATION
}

\author{
Anatoli Djanatliev \\ Reinhard German \\ Computer Networks and Communication Systems \\ University of Erlangen-Nuremberg \\ D-91058 Erlangen, GERMANY
}

\begin{abstract}
Prospective Health Technology Assessment allows early decision making for innovative health care technologies. The main idea is to combine available domain knowledge with advanced simulation techniques in order to predict the effects of medical products and to find bottlenecks and weaknesses within the health system. In our recent publications a hybrid simulation approach with System Dynamics and Agent-Based Modeling has been presented. Hospital workflows have been modeled by state charts within agent behavioral models and have to be instantiated each time an agent is entering a hospital. This paper presents a mechanism to generate agents dynamically from SD models and extends the previously presented hybrid approach by process-oriented Discrete Event Simulation for hospital modeling. It connects processes to health care institutions and not to persons traversing them. Two extended example case studies show potentials for medical decision making using the three simulation paradigms in a common environment.
\end{abstract}

\section{INTRODUCTION}

New technologies are important to support the complex health care delivery and to tackle an increasing demand of health care services in future (Reinhardt 2003, GNYHA 2007). Predicting the effects of such innovations is often like a "look into a crystal ball". In most cases such products have to be inspected by evidence data and by results of early assessment studies (e.g., animal testing), before they can be used in practice. As high quality of health care products is crucial to prevent severe damages of people, this task has to be done very accurately and the regulatory barriers for market launch are hard to break. Furthermore, concurrent perspectives of different stakeholders have to be taken into account. For example patients are expecting efficient health services, insurances are aiming for cost-effectiveness and the health industry is interested in yield maximization primarily. A consequence of this situation is that the development of new products consumes a considerable amount of time and is very cost-intensive. Thus, it can become risky and non-profitable for companies, if an innovation will be rejected by negative assessment results after its development. To achieve an overall acceptance of a new technology and to prepare a product for market launch several decision makers have to be satisfied before. For this reason foresight assessment methods are necessary that help to learn about the impacts of new technologies as early as possible.

Prospective Health Technology Assessment (ProHTA) is a tool that can be applied to support the decision making process. ProHTA is a part of the Centre of Excellence for Medical Technology and is located within the Medical Valley EMN (European Metropolitan Region Nuremberg). This approach uses hybrid simulation techniques to assess new innovative healthcare products early, in particular before the design and development phase has started (Djanatliev et al. 2012). The idea is to optimize the product before its development and to estimate its potentials on a market prospectively. To tackle problems at high 


\section{Djanatliev and German}

abstraction levels (e.g., government perspective) System Dynamics (SD) can be used, at a more detailed and individual level the Agent-Based Simulation (ABS) has been identified to be appropriate.

In our recent publications (Djanatliev and German 2013; Djanatliev et al. 2012) hospital workflows have been modeled by state charts within agent behavioral models and have to be instantiated each time an agent is entering a hospital. This paper presents a mechanism to generate agents from SD models in a dynamic fashion and extends the previously presented hybrid approach by process-oriented Discrete Event Simulation (DES) for hospital modeling where agents are used as entities. In this case processes are connected to health care institutions in hybrid models and not to persons traversing them. Depending on the modeling question at hand all three simulation paradigms can be used and combined, in order to find an adequate modeling detail and sufficient simulation performance. Finally, two extended example case studies show potentials for medical decision making using the three simulation paradigms in a common environment.

\section{RELATED WORK}

The scope of ProHTA relates to the following domain fields in particular: medical decision making, health economic evaluations, health care simulation and multi-paradigm modeling.

In general, it is not easy to make decisions in medicine, however it is important to find common solutions regarding different perspectives. Hence, a manufacturer has to decide together with clinicians and regulatory agencies, if an innovative idea should be implemented or not. This approach helps to prevent from wasting the resources and to develop products that are based on real demand and not only on technical opportunities. A platform to come together in Germany is, e.g., a common conference (ZMT 2011) with all involved players: manufacturers, representatives of insurances, politicians, regulatory agencies, clinicians and so on. Making decisions in health care is usually mapped to policy making, economic issues and process construction steps. Health Technology Assessment (HTA) and Horizon Scanning can be used to study short- and long-term consequences of already developed health care technologies (Sullivan et al. 2009). Analyses of collected data and systematic literature research of medical studies are therefore two most common fields that are currently used to assess health technologies and to support decision makers. The main problem of these tools is that they can only be applied after a product has been developed. Thus, it primarily covers the perspective of policy makers and does not prevent manufacturers from unprofitable investments. A further important field is the domain of health economic evaluations. The main idea is to assess a technology from a financial viewpoint. In order to use a systematic approach for such evaluations, decision-analytic modeling techniques can be applied. Most common tools are decision trees and Markov modeling (Becker, Leidl, and Stollenwerk 2010). Decision trees help to compare treatment alternatives using probability values at the branches and cost values at the leaves. Markov models are using states to represent a health status and probabilities at transitions to reflect different processes (e.g., remission by changing from the state affected to healthy with a certain probability). A problem of such decision-analytic modeling is the complexity when developing large scenarios. This is why DES is used increasingly for health economic evaluations.

There is also significant work in the field of health care simulation and modeling. An example for hospital simulation models was recently presented by Gunal (2012). Brailsford et al. (2009) discussed the problem of stakeholder engagement in health care simulations focusing on problems and proposing guidelines for successful engagements. The scope of ProHTA includes large simulations to inspect new innovations from different perspectives. At high abstraction levels SD can be applied and at low abstraction levels ABS is appropriate. Combining different simulation paradigms in a common simulation environment is a popular topic within recent research publications. Zulkepli, Eldabi, and Mustafee (2012) presented an example application of hybrid simulation for large systems. Brailsford, Desai, and Viana (2010) discussed hybrid simulation using SD and DES in health care. Further issues on hybrid simulation and their application to ProHTA will be outlined in the next section. 


\section{COMBINED SYSTEM DYNAMICS, DISCRETE-EVENT AND AGENT-BASED SIMULATION}

Searching for suitable solutions for medical problems is not an easy task and is often risky or even impossible in the real world. A common technique to guide decision-making in real problems is simulation and modeling which helps to represent systems in abstract models to optimize the health care delivery. There are different approaches that have the power to support such processes. As already mentioned SD, ABS and DES are mainly used in health care and all of them have their legitimation to be applied. The selection of an appropriate method depends on the questions to be answered. Health technology assessments have to be done respecting different perspectives and usually have a large scale which is an important reason to use all simulation paradigms for ProHTA in a hybrid simulation environment. Before we present the hybrid simulation approach of ProHTA, a short introduction to SD, ABS and DES will be given focusing on application in the health care domain.

\subsection{Introduction to Proposed Simulation Paradigms}

Global and "bird's view" scenarios can be modeled by SD following a top-down approach. An example for its use in health care is modeling of nation's health status, separating a population in different dimensions (e.g., age, gender, region) and defining processes by flows and rates (e.g., remission rate, incidence rate) between population crowds that are represented by stocks (e.g., affected, not affected). This kind of continuous simulation represents differential equation systems and helps to inspect the dynamic behavior by changing variables over time. The following equation shows a general calculation process of a stock level $L$ at the time $t+\Delta t$ with $n$ incoming flow rates $\operatorname{In}(t)$ and $m$ outgoing flow rates $O u t(t)$. An approximation sign is used, as rates are not constant and can change within the time interval $\Delta t$ :

$$
L(t+\Delta t) \approx L(t)+\sum_{i=1}^{n}\left(\operatorname{In}_{i}(t) * \Delta t\right)-\sum_{i=1}^{m}\left(\operatorname{Out}_{i}(t) * \Delta t\right)
$$

Classically, SD has no stochastic variability, but many software tools enable a simulation to sample variables by function calls. In most cases System Dynamics models require less data and computing time than more detailed models. Potentials of SD in health care modeling has been presented in Brailsford (2008), a further example can be found in Homer and Hirsch (2005). Simulation at an abstract level can help governments to estimate future trends in order to take precautions. One example would be vaccine reservations in situations of fast disease spreading to keep nation's health status and economic performance.

There are many situations where a more detailed level of simulation is necessary. In scenarios focusing on individual patient treatment steps, micro-simulation approaches at an individual level can be applied. One established tool for this purpose is ABS. According to Macal (2011) the term agent is not yet precisely defined in this context, but the author proposes the following properties and attributes to define "typical agents": an agent is self-directed with an individual behavior, self-contained with attributes and other characteristics, interacts with other agents (social) and an agent has a state that influences its behavior and vice versa. In case of health care modeling, persons (e.g., patients, doctors) can be represented by "typical agents", but it is also possible to model rescue service vehicles and other resources using agents. ABS gives the modeler the possibility to reproduce the reality at a detailed level in a straightforward manner. The main problems of this kind of simulation are typically high data requirements and eventually bad simulation performance (dependent on a scenario).

A further representative of discrete simulation is DES. Using this approach, variables and states change after events had been fired at discrete time points. DES allows to develop models at different abstraction levels. Aggregated scenarios from a strategic viewpoint can be built flexibly, as well as such at more detailed levels. In health care DES is widely used for modeling and optimization of hospital workflows and other processes. An example for this are simulations of waiting times at dedicated stations in a hospital and changes of resource numbers and usage plans. 


\section{Djanatliev and German}

An event can be fired cyclically by a rate, after a time interval has been elapsed, or if certain conditions are true. Heath et al. (2011) presented two different world views for DES. A basic and fundamentally event-oriented world view and a process-oriented world view. According to the authors the latter one is much more common today and is most typically used by commercial DES software packages. The idea is to define processes that can be traversed by entities using a number of different resources. In contrast to agents in ABS, entities are modeled without an individual behavior and are just data objects influencing system's decision processes. In order to extend our previous SD and ABS hybrid simulation approach for ProHTA we use the process-oriented DES for hospital modeling. This will be presented in more detail in the next section.

According to the complexity and the heterogeneity of medical processes, many established modeling and simulation techniques are not sufficiently powerful. Thus, using different methods to answer a common question is getting popular in current health care modeling and simulation research. Macal (2010) presented a formal specification of SD and ABS in order to derive equivalent ABS models. Three different formats to combine SD with DES in a common simulation environment has been introduced by Chahal and Eldabi (2008). In ProHTA we consider the process environment format in particular. The hybrid simulation methodology is not precisely defined yet (Heath et al. 2011), but in most cases a combination of continuous and discrete paradigms can be seen as hybrid. In contrast to this, we consider the multi-paradigm modeling as a combination of different modeling and simulation approaches without an explicit distinction between continuous and discrete. This allows more focusing on problem solving and not spending time on formalisms. However it is important to take into account how continuous and discrete models are coupled in hybrid models, as it plays also an important role in multi-paradigm modeling.

\subsection{Multi-Paradigm Simulation Framework for Health Technology Assessments}

ProHTA is a decision support tool that uses multi-paradigm simulation to evaluate large health technology innovation scenarios from different perspectives. A first approach for this purpose has been presented in Djanatliev et al. (2012). To tackle the complexity several generic and scenario specific modules have been defined. Further important units in this context are in particular GeoDynamics (GD), Data Component (DC) and Error Injector (EI). GD gives the possibility to model regions and districts separately and uniquely (e.g., with individual age distributions). DC allows to perform simulation input modeling at a central location. It prevents the modeler to search for parameters in large models when performing changes. A further advantage is data preparation. Thus, data can be loaded on startup or dynamically on runtime directly from resources (e.g., files, data bases) and can be prepared for expecting modules. EI is necessary to develop models for error injections (e.g., wrong resource usages). A current set of modules and detailed definitions of the modular units can be found in Djanatliev and German (2013). To reduce the model development time in future scenarios, the components have well-defined interfaces and are placed into a common toolbox in order to prepare them for reuse. Once validated, the modules must only be checked within the context of new scenarios and not internally.

The implementation of this approach is done using the commercial tool AnyLogic 6 (XJ Technologies Company Ltd. 2013). This software package provides possibilities to develop multi-paradigm models in a common environment and enables to enrich the implementation by additional Java code. This makes the tool very flexible and appropriate for large hybrid modeling. As implementation is not a focus of this paper, a more detailed description of these issues can be found in Djanatliev and German (2013). It provides also information on architectural aspects of hybrid health technology assessment models using a dedicated LevelBased Architecture (LBA) and an inter-module communication mechanism with a centralized instantiation approach. 


\subsubsection{Modeling Hospital Workflows by Process-Oriented DES}

In the previous ProHTA hybrid simulation approach hospital workflows are modeled by state charts within behavioral models of the patient agent type. When an agent is entering the treatment phase state a new active object of a hospital process state chart will be instantiated and the agent traverses through its process states. A drawback of this method is a replicated number of equal hospital process objects and an imprecise logical belonging, because hospital workflows are logically connected to institutions and not to patients traversing them.

As mentioned previously, hospital workflows can be comfortably modeled by DES. Particularly, the process-oriented worldview (Heath et al. 2011) is a perfect possibility to represent such processes. Viana et al. (2012) presented an example of multi-paradigm simulation with DES used for eye clinic modeling. To take profit from already established DES usage for modeling processes we extended the SD, ABS approach of ProHTA by DES. This possibility allows not only to develop individual processes for different hospitals, it provides a chance to model any kind of workflows in the same manner.

Generic modules of ProHTA are usually modeled independently from each other by applying different simulation paradigms. In most cases LBA is used and the interfaces between the components are defined in a way where it is not important which simulation paradigm is behind (Djanatliev and German 2013). Nevertheless, there are some important recurrent modeling processes (best cases) that are depicted in Figure 1. Modeling of ProHTA studies follows a top-down approach. One starts by preparing a considered population at a high abstraction level using population dynamics and disease dynamics SD modules. Once an appropriate population is found, ABS and/or DES are used to go into detail. How to decide when agents shall be generated and when entities are appropriate will be explained shortly in section 3.2.3. The generation process itself will be the focus of the next section.

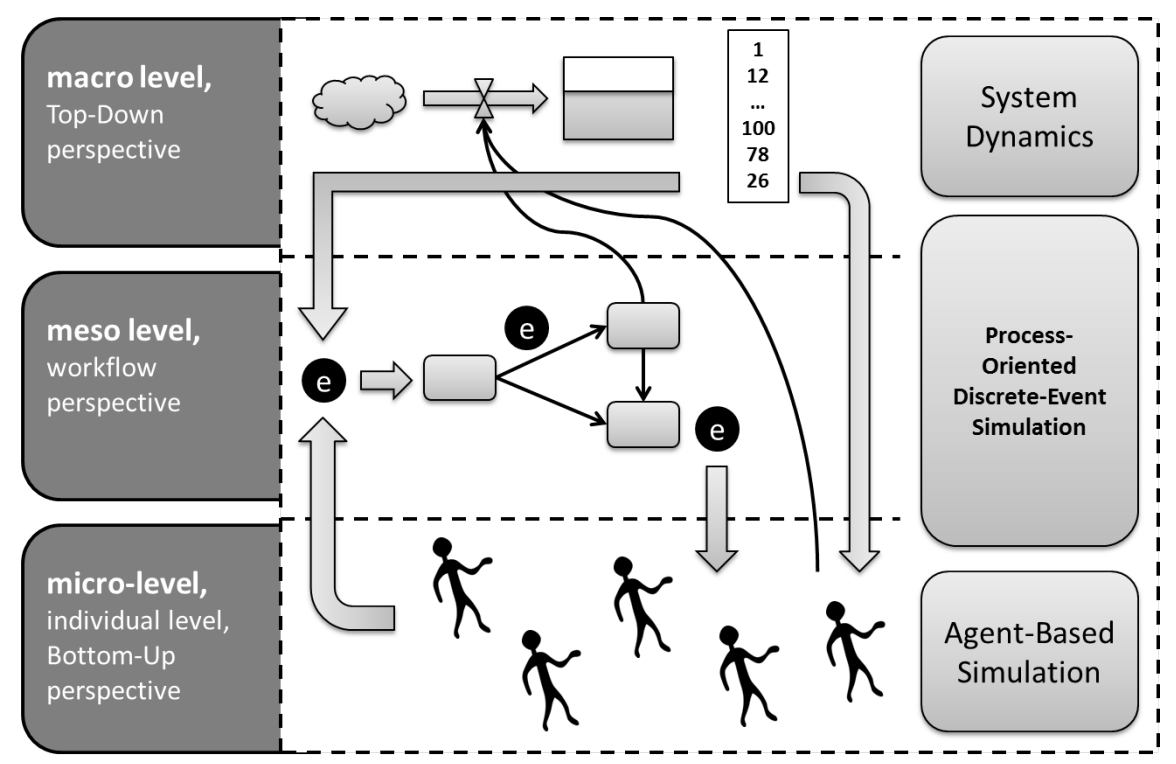

Figure 1: Overview of important ProHTA processes between abstraction levels.

When an agent has to traverse workflows, its behavioral state chart will be paused in the state treatmentPhase and the agent will be packed together with a temporarily created health record into the entity type AgentEntity. While this entity traverses a workflow, decisions can be made using the attributes of the containing agent and filling the health record for further decisions. For example, a diagnosis result is filled into the health record and a therapy decision can be made dependent on this information. After finishing the process, the agent will be unpacked from the corresponding entity and its 
attributes and variables have to be adjusted with respect to the health record. Finally, the entity and the health record object will be removed and the behavioral state chart of the agent will be resumed. This modeling approach allows to accelerate simulation performance while keeping full flexibility.

Technically, the implementation can be achieved by the AnyLogic EnterpriseLibrary which is appropriate for this purpose. It provides the most important required elements, such as decision, delay or resource pool and can flexibly be customized by Java coding.

\subsubsection{Generating Agents and Entities from SD Environment}

According to large population numbers in usual ProHTA studies, representing each person by agents in ABS is impossible. However, smaller crowds are in most cases enough to answer certain questions (e.g., only affected persons). At this point, we can take advantages from a multi-paradigm simulation. The affection process can be modeled by SD at a high level. The number of affected persons $A f f$ can be calculated in a stock using equation (1) with incidence as inflow rate and mortality of affected as outflow rate (simplified representation).

$$
\text { Aff }(t+\Delta t) \approx A f f(t)+\text { incidenceRate } * \Delta t-\text { mortality } * \Delta t
$$

In most cases it is important to generate affected crowds which can be differentiated into different dimensions to parametrize agents/entities afterwards (e.g., age, gender). In this case dimensioned stocks and flows of AnyLogic can be applied. Instead of modeling replicated SD models with different rate values, the tool allows to use vectors and matrices of rates to define flows and the resulting stock is represented by the same dimension type containing the different corresponding level values. Figure 2 presents an example SD model of this process.

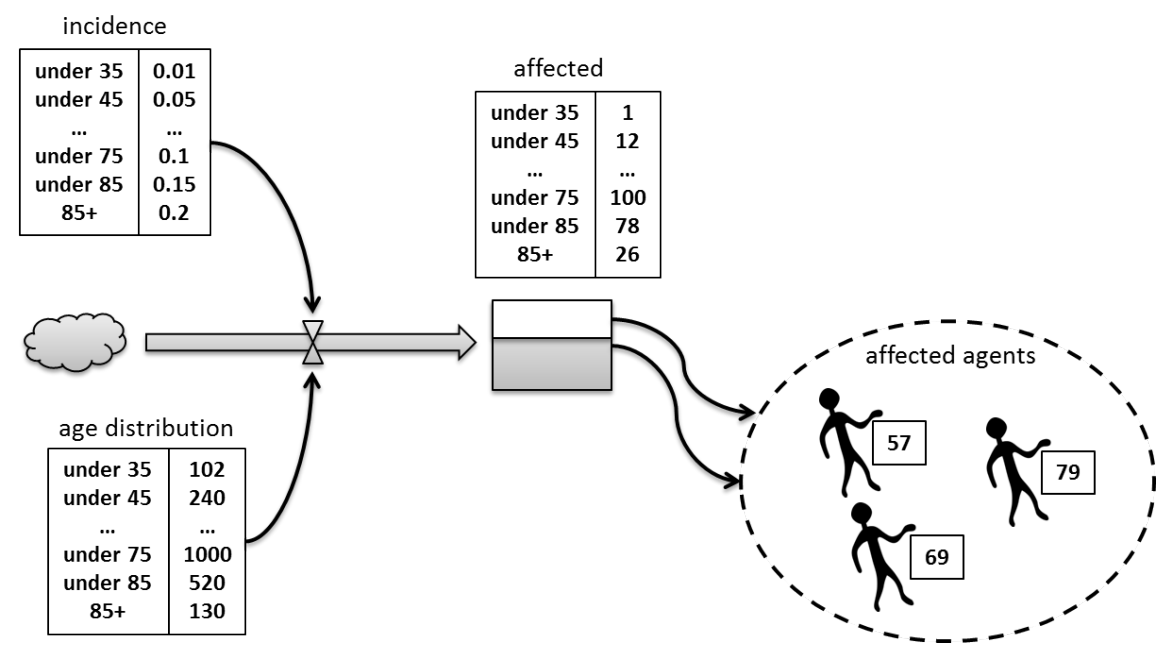

Figure 2: Example: Generating agents/entities from SD model level.

In this case a vector of affected persons can be calculated by multiplying the age specific incidence values and the corresponding age distribution which is calculated in parallel by the demographic component. The resulting stock is a vector containing a dedicated number of affected persons within different age groups. These numbers will now be used to generate individuals. Agents/entities can die after their instantiation, so the mortality process is modeled in stochastic ABS/DES models. This approach can be applied individually for each district to use district specific distributions and parameters.

The generation process itself starts by pick-ups of the calculated population numbers. Due to an observation time interval (e.g., month) the vector with persons to be represented by agents/entities is filled 


\section{Djanatliev and German}

by the just described approach. After the pick-up event fires, the vector values are set to 0 and new dynamic events will be scheduled in the next time interval uniformly for each picked-up agent/entity to distribute the agent generation by time. When this event is fired a new individual is produced. The attributes are sampled by the picked-up information. In our example, the current age can be sampled using the age distribution within the dedicated age group.

\subsubsection{Further General Modeling Aspects}

Entities are usually more lightweight than agents. Hence, when an individual behavior is not absolutely necessary it is recommendable to generate entities using the type PersonEntity for workflow traversing. Otherwise, agents have to be generated and will be transformed to entities using the type AgentEntity when a hospital process has to be traversed. As previously mentioned, the agent will be reactivated when a hospital workflow has been finished.

Until now, the described generation process shows merely the direction from abstract SD models to more detailed ABS/DES ones. However, in multi-paradigm environments the reverse coupling is also an important aspect. Usually, SD models are controlled by external parameters and auxiliaries, but in hybrid models DES workflows and agent behavior can also change the dynamics (e.g., flow rates) at high abstraction levels. An example for this is the calculation of the case fatality rate used in an SD component. Thus, this value will be adjusted dynamically when agents/entities are "dying" according to a (stochastic) disease specific mortality process of individual agents/entities.

In Djanatliev and German (2013) a possibility to collect agents with similar behavior in so-called Post Disease Containers has been presented. The idea is to perform set calculations instead of identical routines for each agent in order to increase the simulation performance. An example for this would be the combination of all agents being in the first year after a considered disease with the same index of disability. Thus, the number of agents can be used to calculate costs or a subset can be selected to traverse annual post disease processes (if other attributes are not important to perform this selection).

\section{DECISION MAKING USE-CASES}

As presented in Djanatliev et al. (2012) ProHTA can be used to solve two kinds of problems. On the one hand what-if questions can be answered to learn about the impacts of new innovative technologies, otherwise new ideas and technologies can be found by answering to how-to questions (e.g., how to reach desired effects).

The presented methodology in this paper can be applied to support decision making within the scope of ProHTA by focusing on these two types of questions and considering different perspectives. For example, simulations can be run prospectively to inform regulatory agencies, as well as to analyze chances for a market launch of a new technology. This helps managers of companies to decide, weather a new product shall be developed or not. In case of negative simulation results, an innovation can be optimized, or the project can be rejected to prevent unprofitable investments.

Main process steps of ProHTA have been presented in Gantner-Bär et al. (2012) including in particular, domain knowledge collection by an interdisciplinary team of experts and definition/implementation of different simulation scenarios for problem solving. The first type of questions can be answered by comparing a reference scenario to an innovative one with already included innovation(s). Evaluations aiming at how-to questions can be performed by varying parameters until desired effects can be reached. In a second step a new technology idea can be derived that is able to fulfill the configured parameter values.

Although all parameters are eligible for optimization of output metrics, the most important ones are sensitivity and specificity values of different diagnostic interventions. A detailed definition of these two terms can be found in Akobeng (2007). Thus, sensitivity is a proportion of affected people with a positive diagnostic result and specificity gives the proportion of healthy people with a negative test result. In case of 
answering to how-to questions, this parameters can be varied in order to find a proper solution. Afterwards, a new technology has to be invented that can fulfill an adjusted configuration.

Two examples for decision making supported by ProHTA are presented in the following. We do not concentrate on complete study descriptions and detailed validation steps, as it would be beyond the scope of this paper, but on practical use of the presented methods. Hence, the examples will be described only shortly. Example 1 is primarily focused on what-if questions within the stroke therapy and example 2 shows a practical use-case for answering to how-to questions.

\subsection{Example 1: Evaluation of Mobile Stroke Units}

In our recent publications a use case with Mobile Stroke Units (MSUs) has been presented (e.g., Djanatliev et al. 2012). Stroke is a major reason for disability of people and consumes a high amount of costs for rehab and care. According to population aging and increasing life expectancy, stroke therapy offers potentials for new products and application fields for technology assessments. Thrombolysis is an effective therapy, but is very time-critical. To tackle this problem, new specialized vehicles (MSUs) are in focus of the current research efforts. This technology helps to perform important diagnostic steps on-site at patient's location in order to start with the therapy immediately.

\subsubsection{Modeling Remarks}

In our model onset-to therapy decision time is used to sample a Barthel-Index (Quinn, Langhorne, and Stott 2011) which serves as an indicator for disability of people. Figure 3 depicts two ABS/DES model examples. On the left hand side the main behavioral state chart of the agent type person is presented. After an agent has been generated from the SD environment (as presented in section 3.2.2) the state chart will be started by entering the cognition phase. Dependent on the time of occurrence (e.g., night, day) it takes a certain time interval until a person recognizes an affection and calls the emergency service. Thereafter, the agent enters the pre-treatment state and a new active object including a rescue service process will be instantiated.
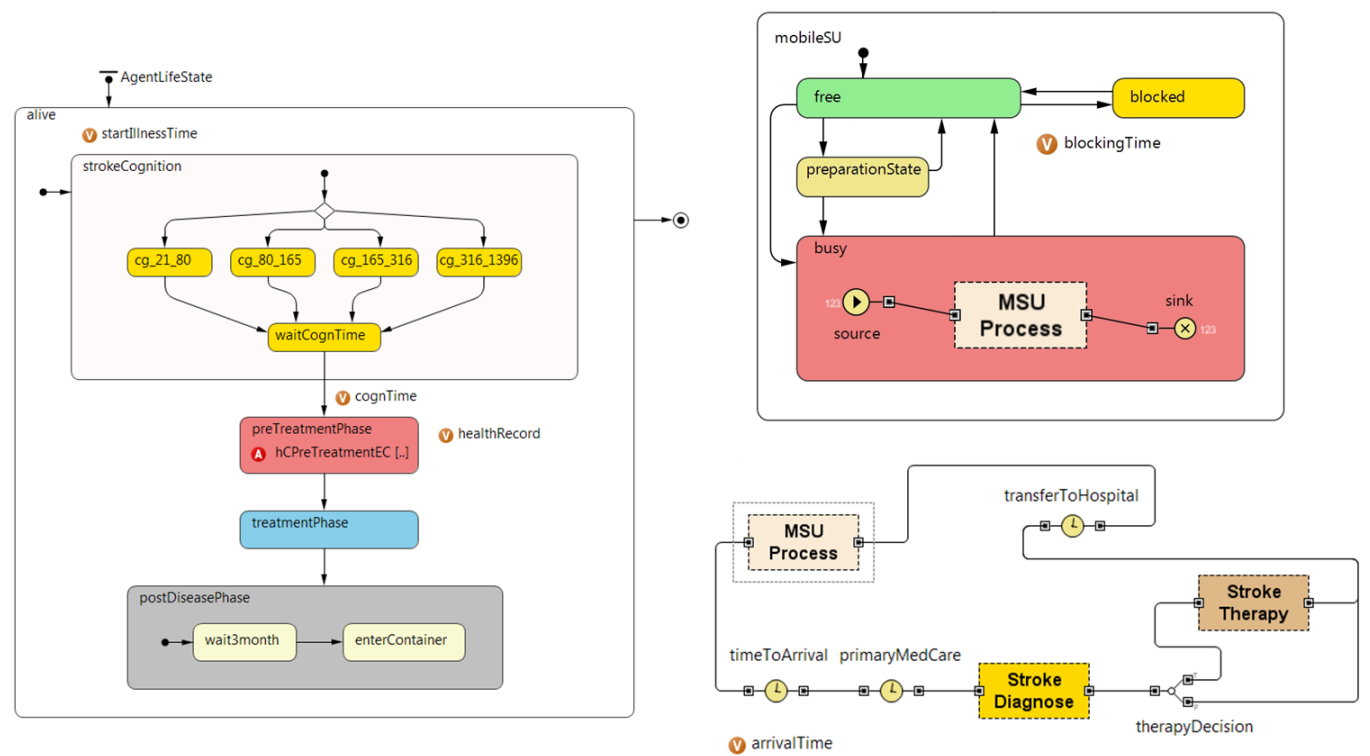

Figure 3: Example ABS/DES models: on the left hand the main behavioral state chart of persons is depicted, on the right hand a state chart of MSUs can be found and the top-level diagram of an MSU process. 
The dispatcher takes a first diagnosis using the sensitivity of the short interview. In case of stroke decision, all agents that are inside a radius of SSU or MSU will be reached by sampling a time from a certain time distribution that can be configured before the simulation has been started. The patient will be transported to the next Stationary Stroke Unit (SSU) by a normal rescue vehicle, if its location is inside a predefined radius of an SSU. In cases when the location is inside the radius of a free MSU, the innovative vehicle will be sent to the affected person. Dependent on the distances to the next free MSU or SSU, patients that are outside of all radii will be picked-up by the next free MSU or will be transported to the next SSU.

MSUs are modeled by an own agent type. The right hand state chart of Figure 3 represents the behavioral states of this vehicle. When MSUs are used by mistake (reproduced by the Error Injector component) the blocked state will be activated, otherwise the vehicle enters the busy state and is not available for dispatchers within this time period. Afterwards, the hierarchical process diagram of the MSU process will be traversed by an entity of the type MSUAgentEntity containing the target person and the corresponding MSU agents. The top-level diagram of the MSU process can be found at the lower right side of Figure 3.

Figure 4 shows the animation screenshot of an example simulation run for the area of Berlin. Affected persons are represented by dots that are located at patient's home location. The color of the dots represents the current state of an agent. Cognition is represented by the yellow color, agents in pre-treatment are colored in red, blue dots represent agents in hospitals and grey ones are people within the post-stroke phase. MSUs are represented by vehicle icons. Moving MSUs colored in blue mean that a vehicle is busy. Otherwise, the radius is visible when an MSU is free. Further details concerning the scenario and detailed descriptions of the model can be found in Djanatliev and German (2013).

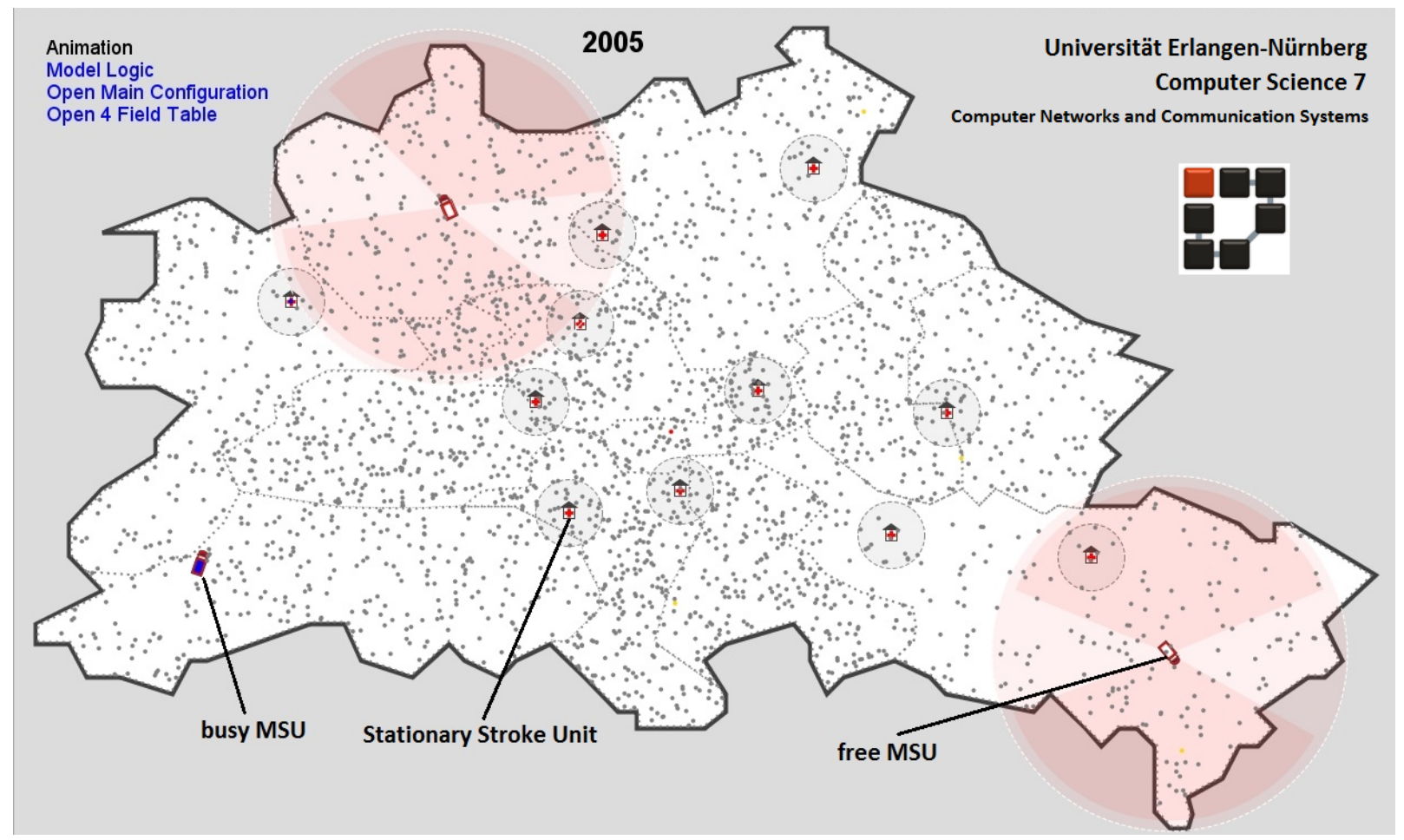

Figure 4: Screenshot of the Mobile Stroke Unit animation containing affected persons (dots), SSUs and MSUs. 


\subsubsection{Answering what-if Questions}

There are many possible scenarios to evaluate MSUs. Generally, output metrics have to be analyzed to inspect the effects of this innovation. An example result is depicted in Figure 5 and shows a distribution of onset-to treatment times with/without Mobile Stroke Units. Considering locations of persons and MSUs it is possible to define several what-if scenarios. An example therefore is to find optimal positions for MSUs regarding shorter time results. To perform such evaluations a positioning tool can be used that was implemented for this purpose. One important result is the proportion of people in different onset-to treatment time groups. First results show that a higher significant number of people reaching the therapy within the most favorable time interval of 0-90 minutes can be counted when the same number of MSUs are better distributed as in scenarios when the vehicles are located near to each other. As this example is only used to present decision making by answering to what-if questions, a more detailed study focusing on positioning evaluations will be presented in an upcoming publication.

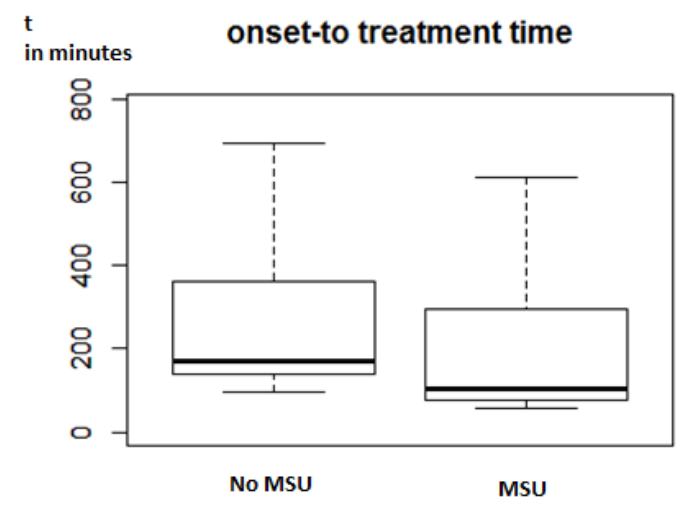

Figure 5: Distributions of the onset-to treatment time with/without using Mobile Stroke Units.

\subsection{Example 2: Configuration of Innovative Markers for Prostate Cancer Screening}

Prostate Cancer (PC) is a disease that mainly occurs at men that are more than 45 years old. Usually, it is important to detect available affections as early as possible to keep chances for remission. PC is in most cases painless, so it is important to perform preventive screenings after reaching the age of 45 years in order to detect an affection at an early stage.

Most common diagnostic interventions to detect prostate cancer are Digital-Rectal Examination (DRE), Prostate-Specific Antigen (PSA) and Biopsy. The latter one is usually an unpleasant and painful examination. Furthermore, there is a probability that complications will remain after executions of this intervention. To save costs for unnecessary examinations and to prevent healthy people from side effects, it is important to have an effective diagnosis process. Usually, the PSA test is applied after DRE and has low specificity values. This means that many not affected men will have positive test results and will be forwarded to biopsy. In order to prevent this situation, a new intervention is necessary which can reduce the costs and the number of not affected men having complications after biopsy.

This how-to question can be answered by ProHTA as follows. We invent a new abstract intervention that will be used after PSA and start to "play" with sensitivity, specificity and additional costs-perusage parameters. Using the number of avoided biopsies and comparing the cost-effectiveness, a suitable configuration can be found. The last step will be to find a new technology that can be developed regarding the obtained cost-per-usage and sensitivity/specificity values to fill the gap of the abstract innovation. An appropriate solution for this scenario can be the implementation of an innovative marker matching the expected output metrics. 


\section{CONCLUSION}

Decision making in the field of health technology assessment is not a simple task and is important for different stakeholders, particularly for health industry companies. ProHTA is a tool that allows assessments of new innovations before a product has been developed and even designed. Using this approach a product can be optimized prospectively and new potential product ideas can be derived.

In this paper a multi-paradigm simulation method has been presented using SD for simulations at a high abstraction level and ABS/DES at an individual level in a common simulation environment. In particular, process-oriented DES can be used to represent hospital processes that can be traversed by entities. Furthermore, a generation process of individual objects from SD models has been described. Entities for DES can be generated directly, if an individual behavior is not necessary, otherwise agents will be produced for ABS. Hospital workflows can be traversed by agents after they had been packed into a dedicated entity type together with a temporary health record object. Afterwards, the agent object will be unpacked and the behavioral state chart will be resumed by exiting from the treatment phase state.

Finally, the most important steps of the decision making process using ProHTA have been presented. Two examples show how a new innovative technology can be evaluated prospectively (what-if) and how new ideas can be derived by parameter variations (how-to). Both use-cases have been validated with our experts. We performed structured validation steps for each module first and proceeded then towards an overall model validation (Djanatliev and German 2013). In general, it was necessary to make the model transparent to domain experts by several tests (e.g., sensitivity analyses) in order to gain an overall credibility.

\section{ACKNOWLEDGMENTS}

On behalf of the ProHTA Research Group. We gratefully acknowledge P. Kolominsky-Rabas as well as the whole ProHTA Research Group for support on development and validation of the two use-cases. ProHTA is funded by the German Federal Ministry of Education and Research (BMBF) as part of the National Cluster of Excellence Medical Technology - Medical Valley EMN (Project grant No. 01EX1013B).

\section{REFERENCES}

Akobeng, A. K. 2007. "Understanding diagnostic tests 1: sensitivity, specificity and predictive values". Acta Paediatrica 96 (3): 338-341.

Becker, C., R. Leidl, and B. Stollenwerk. 2010. "Decision-Analytic Modelling in Economic Evaluation". Gesundheitsökonomie \& Qualitätsmanagement 15 (05): 260-264.

Brailsford, S. C. 2008. "System Dynamics: What's in for Healthcare Simulation Modelers". In Proceedings of the 2008 Winter Simulation Conference, 1478-1483. Winter Simulation Conference 2008.

Brailsford, S. C., T. Bolt, C. Connell, J. H. Klein, and B. Patel. 2009. "Stakeholder Engagement in Health Care Simulation". In Proceedings of the 2009 Winter Simulation Conference, 1840-1849.

Brailsford, S. C., S. M. Desai, and J. Viana. 2010. "Towards the Holy Grail: Combining System Dynamics and Discrete-Event Simulation in Healthcare". In Proceedings of the 2010 Winter Simulation Conference, 2293-2303.

Chahal, K., and T. Eldabi. 2008. "Applicability of Hybrid Simulation to Different Modes of Governance in UK Healthcare". In Proceedings of the 2008 Winter Simulation Conference, 1469-1477. Winter Simulation Conference 2008.

Djanatliev, A., and R. German. 2013. "Large Scale Healthcare Modeling by Hybrid Simulation Techniques using AnyLogic". In Proceedings of the 6th International ICST Conference on Simulation Tools and Techniques.

Djanatliev, A., P. Kolominsky-Rabas, B. M. Hofmann, A. Aisenbrey, and R. German. 2012. "Hybrid Simulation Approach for Prospective Assessment of Mobile Stroke Units". In Proceedings of the 2nd International Conference on Simulation and Modeling Methodologies, Technologies and Applications, 357-366. 
Djanatliev, A., P. Kolominsky-Rabas, B. M. Hofmann, and R. German. 2012. "Hybrid simulation with loosely coupled system dynamics and agentbased models for prospective health technology assessments". In Proceedings of the 2012 Winter Simulation Conference.

Gantner-Bär, M., A. Djanatliev, H. U. Prokosch, and M. Sedlmayr. 2012. "Conceptual Modeling for Prospective Health Technology Assessment". In Proceedings of the XXIV Conference of the European Federation for Medical Informatics.

GNYHA 2007. "Health Care Demand Rising, Population Aging: The Imperative to Increase Physician Supply". Health Care News In-Depth.

Gunal, M. M. 2012. "A Guide for Building Hospital Simulation Models". Health Systems 1 (1): 17-25.

Heath, S. K., S. C. Brailsford, A. Buss, and C. M. Macal. 2011. "Cross-Paradigm Simulation Modeling: Challenges and Successes". In Proceedings of the 2011 Winter Simulation Conference, 2788-2802. Winter Simulation Conference 2011.

Homer, J. B., and G. B. Hirsch. 2005. "System Dynamics Modeling for Public Health: Background and Opportunities". American Journal of Public Health (Vol 96, No. 3): 452-458.

Macal, C. M. 2010. “To Agent-Based Simulation from System Dynamics". In Proceedings of the 2010 Winter Simulation Conference, 371-382.

Macal, C. M. 2011. "Introductory Tutorial: Agent-Based Modeling and Simulation". Proceedings of the 2011 Winter Simulation Conference:1456-1469.

Quinn, T. J., P. Langhorne, and D. J. Stott. 2011. "Barthel Index for Stroke Trials: Development, Properties, and Application". Stroke 42 (4): 1146-1151.

Reinhardt, U. E. 2003. "Does The Aging Of The Population Really Drive The Demand For Health Care?". Health Affairs 22 (6): 27-39.

Sullivan, S. D., J. Watkins, B. Sweet, and S. D. Ramsey. 2009. "Health Technology Assessment in Health-Care Decisions in the United States". Value in Health 12:S39-S44.

Viana, J., S. Rossiter, A. A. Channon, S. C. Brailsford, and A. Lotery. 2012. "A Multi-Paradigm, Whole System View of Health and Social Care for Age-Related Macular Degeneration". Proceedings of the 2012 Winter Simulation Conference.

XJ Technologies Company Ltd. 2013. "AnyLogic - www.xjtek.com - Accessed April 12".

ZMT 2011. "Zukunftskonferenz für Medizintechnik - http://www.zukunftskonferenz-medizintechnik.de".

Zulkepli, J., T. Eldabi, and N. Mustafee. 2012. "Hybrid Simulation For Modeling Large Systems: An Example of Integrated Care Model". Proceedings of the 2012 Winter Simulation Conference.

\section{AUTHOR BIOGRAPHIES}

ANATOLI DJANATLIEV received a diploma in computer science in 2009. Afterwards, he worked as software engineer within the domain of statistical and model-based software testing. Currently he is a research assistant at the Department of Computer Science 7, University of Erlangen-Nuremberg. His research interests include prospective assessments of health technology by simulation, agent-based simulation and hybrid simulation modeling. His email address is anatoli.djanatliev@fau.de.

REINHARD GERMAN received a diploma in computer science in 1991, the PhD degree in 1994, and the habilitation degree in 2000 from the Computer Science Department, Technical University of Berlin. Thereafter, he joined the Department of Computer Science at the University Erlangen-Nuremberg. First, he was an associate professor (system simulation), then he became a full professor in 2004 (computer networks and communication systems), and served as head of the department and as dean of the faculty of engineering. His research interests include performance and dependability analysis of networked systems based on numerical analysis, network calculus, discrete-event simulation, measurements, and testing. Vehicular communications, smart energy systems and healthcare constitute major application domains. His e-mail is german@cs.fau.de. 